\title{
Isothermal Environmental Heat Energy Utilization by Transmembrane Electrostatically Localized Protons at the Liquid- Membrane Interface
}

James Weifu Lee*

Cite This: ACS Omega 2020, 5, 17385-17395

Read Online

ABSTRACT: This study employing the latest theory on transmembrane electrostatic proton localization has now, for the first time, consistently elucidated a decadeslongstanding bioenergetic conundrum in alkalophilic bacteria and more importantly discovered an entirely new feature: isothermal environmental heat utilization by electrostatically localized protons at the liquid-membrane interface. It was surprisingly revealed that the protonic motive force (equivalent to Gibbs free energy) from the isothermal environmental heat energy utilization through the electrostatically localized protons is not constrained by the overall energetics of the redox-driven proton pump system because of the following: (a) the transmembrane electrostatically localized protons are not free to move away from the membrane surface as a protonic capacitor feature; (b) the proton pumps embedded in the cell membrane extend beyond the localized proton layer apparently as an asymmetric property of the biological membrane; and (c) the protonic inlet mouth of the ATP synthase that accepts protons

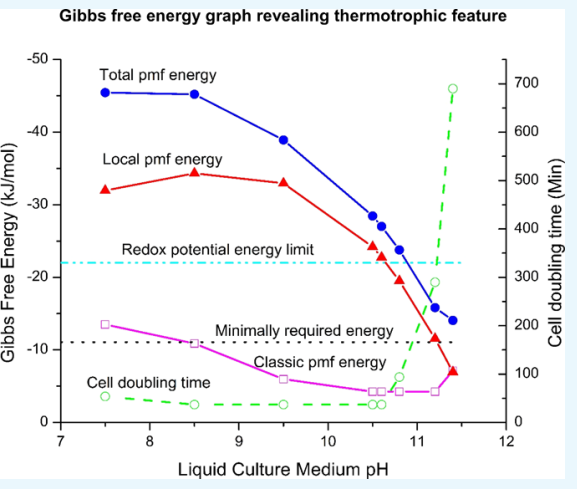
is located within this layer as another natural property of the asymmetric biological membrane. This work has now, for the first time, shown a novel thermotrophic feature where biological systems can isothermally utilize environmental heat energy through transmembrane electrostatically localized protons to help drive ATP synthesis.

\section{INTRODUCTION}

Environmental heat is the dissipated form of heat energy, also known as latent (hidden) heat energy or the temperaturedependent molecular thermal motion energy in the environment. In the past, for centuries, it had been believed that environmental heat (the dissipated form of heat energy) in any mass medium could not be utilized to do useful work unless there is a temperature difference. ${ }^{1}$ That classic belief is now shattered by the new discovery reported here that transmembrane electrostatically localized excess protons at the liquid water-membrane interface can isothermally utilize their molecular thermal motions to do useful work in driving ATP synthesis at the biophysical molecular scale.

This is a groundbreaking scientific discovery also for the field of life sciences where it has been commonly believed that biological systems can use only chemical energy and/or light energy but not the environmental heat (the dissipated form of heat energy). Consequently, based on their sources of energy, the organisms on Earth were generally classified or believed only as two types: chemotrophs and phototrophs. This classic belief is now also shattered by the discovery reported here, which through a bioenergetics elucidation study of alkalophilic bacteria Bacillus pseudofirmus ${ }^{2-4}$ with the Lee transmembrane electrostatic proton localization theory ${ }^{5-9}$ surprisingly revealed that environmental heat can be isothermally utilized through electrostatically localized protons at a liquid-membrane interface to help drive ATP synthesis. Naturally, this raises an important scientific question: Do proton-coupling bioenergetic chemotrophs also have a thermotrophic feature? The answer to this question is now positive.

The story here began with the modern development of bioenergetics including Peter Mitchell's chemiosmotic theory. ${ }^{10-12}$ Mitchell's central bioenergetics equation has entered into many textbooks, ${ }^{13-16}$ which is expressed as the protonic motive force ( $\mathrm{pmf}$ ) across a biological membrane that drives protons through the ATP synthase; It is

$$
\mathrm{pmf}=\Delta \psi-\frac{2.3 R T}{F} \Delta \mathrm{pH}
$$

Here, $\Delta \psi$ is the electrical potential difference (which is defined as positive ( $p$ ) phase minus negative ( $n$ ) phase and is usually positive $\mathrm{e}^{16-18}$ ) across the membrane; $R$ is the gas constant; $T$ is the absolute temperature; $F$ is Faraday's constant; and $\Delta \mathrm{pH}$ is defined as the $\mathrm{pH}$ in the bulk $\mathrm{p}$ phase minus the $\mathrm{pH}$ in the bulk $\mathrm{n}$ phase. ${ }^{15,16}$ In that framework, the protons are

Received: April 17, 2020

Accepted: June 26, 2020

Published: July 9, 2020 
Table 1. B. pseudofirmus OF4 Measured Properties (Data of $\mathrm{pH}_{\mathrm{pB}}, \mathrm{pH}_{\mathrm{nB}}$, and $\Delta \psi$ from Ref 23) and Calculated Quantities Using Eqs $5-7^{a}$

$\begin{array}{lcccccccc}\mathrm{pH}_{\mathrm{pB}} & \mathrm{pH}_{\mathrm{nB}} & \Delta \psi(\mathrm{mV}) & {\left[\mathrm{H}_{\mathrm{L}}^{+}\right]^{0}(\text { molar})} & \text { exchange reduction factor } & {\left[\mathrm{H}_{\mathrm{L}}^{+}\right](\text {molar })} & \text { local pmf }(\mathrm{mV}) & \text { classic pmf }(\mathrm{mV}) & \text { total pmf }(\mathrm{mV}) \\ 7.5 & 7.5 & 140 & 1.92 \times 10^{-2} & 1.50 & 1.28 \times 10^{-2} & 331 & 471 \\ 8.5 & 7.7 & 160 & 2.19 \times 10^{-2} & 6.53 & 3.35 \times 10^{-3} & 356 & 113 & 469 \\ 9.5 & 7.5 & 180 & 2.46 \times 10^{-2} & 128 & 1.93 \times 10^{-4} & 342 & 42 & 404 \\ 10.5 & 8.2 & 180 & 2.46 \times 10^{-2} & 4.41 \times 10^{4} & 5.58 \times 10^{-7} & 251 & 295 \\ 10.6 & 8.3 & 180 & 2.46 \times 10^{-2} & 9.96 \times 10^{4} & 2.47 \times 10^{-7} & 236 & 44 & 280 \\ 10.8 & 8.5 & 180 & 2.46 \times 10^{-2} & 5.85 \times 10^{5} & 4.21 \times 10^{-8} & 202 & 44 & 246 \\ 11.2 & 8.9 & 180 & 2.46 \times 10^{-2} & 3.68 \times 10^{7} & 6.70 \times 10^{-10} & 120 & 44 & 164 \\ 11.4 & 9.6 & 180 & 2.46 \times 10^{-2} & 3.96 \times 10^{8} & 6.21 \times 10^{-11} & 72 & 146\end{array}$

${ }^{a}$ The cation concentrations and proton exchange equilibrium constants are from Table 2 with the temperature $T=298 \mathrm{~K}$. The "local" pmf is the last term in eq 5, while the first two terms of eq 5 give the "classic" Mitchellian pmf (eq 1).

considered as solutes, like sugar molecules in water, that are delocalized, existing everywhere in the two bulk aqueous phases. Consequently, the Mitchellian view of bioenergetics is that the ATP synthase is coupled to the redox proton pumps via the bulk phase-to-bulk phase pmf across the biological membrane. The chemiosmotic theory was a significant milestone in the historical development of modern bioenergetics; its significance to the history of the field could hardly be overstated.

However, we now know that the textbook Mitchellian pmf equation (eq 1) is not entirely correct and thus should be revised. ${ }^{6,7,9,19,20}$ The most well-established observations that disagree with the Mitchellian pmf equation are in alkalophilic bacteria, such as B. pseudofirmus. ${ }^{2-4}$ The alkalophilic bacteria keep their internal $\mathrm{pH}$ at about 2.3 units more acidic than the ambient bulk $\mathrm{pH}$, while $\Delta \psi$ is about $180 \mathrm{mV}^{21-23}$ The use of the Mitchellian pmf equation (eq 1) in this case would yield a pmf value so small ( $44 \mathrm{mV}$ at $T=298 \mathrm{~K}$ ) that for decades has clearly failed to explain the energetics as to how these organisms are able synthesize ATP. ${ }^{24-26}$

Furthermore, the classic Mitchellian chemiosmotic theory apparently has also failed to address the legitimate question as to what extent the proton-coupling pathway for producing ATP is delocalized throughout the bulk aqueous volume or localized at the membrane surface since it was first raised in 1961 by Williams. ${ }^{27-34}$ A number of well-documented observations, such as the localized proton-coupling characteristics observed in the "low salt"-treated thylakoids of the Chiang-Dilley experiment $^{35}$ and the $\Delta \mathrm{pH}$ surface component of pmf in ATP synthesis of mitochondria, ${ }^{36}$ could not be explained by the delocalized proton view of the classic Mitchellian chemiosmotic theory. The newly reported lateral $\mathrm{pH}$ gradient as elegantly probed with bioengineered fluorescent proteins as $\mathrm{pH}$ indicators between OXPHOS complex IV and $\mathrm{F}_{0} \mathrm{~F}_{1}$ ATP-synthase in folded mitochondrial membranes ${ }^{37}$ also implicates a need for "a modification to Peter Mitchell's chemiosmotic proposal.” A recent biomimetic study ${ }^{38}$ points to a similar localized proton phenomenon.

Recently, as summarized briefly below, the author developed a transmembrane electrostatic proton localization theory ${ }^{5,6,8,9,39}$ that elucidates how free excess protons in an aqueous medium separated by an impermeable membrane alone can be localized spontaneously at the liquid-membrane interface. Moreover, a newly developed pmf equation by modifying eq 1 to account for localized protons at a liquid-membrane interface was introduced and shown to result in a large enough pmf for the synthesis of ATP in alkalophilic bacteria. 6,40

Our previous study ${ }^{7,9}$ has clarified that neither the GouyChapman theory ${ }^{41}$ nor the Debye length concept could be applied to estimate the thickness of the transmembrane electrostatically localized excess protons at the liquidmembrane interface here because the classic equations used in calculating the Debye length are defined only for chargebalanced solutions including 1:1 electrolyte (such as $\mathrm{NaCl}$ ) solutions. ${ }^{42}$ Since the membrane is a type of insulator film (not an electrode), the excess proton layer at the water-membrane interface is likely to be a special monolayer (with a thickness probably about $1 \mathrm{~nm}$ ) but definitely not an "electric double layer" as that of a typical electrode/electrolyte solution system as expected by the Gouy-Chapman theory. ${ }^{41}$ The current understanding of an excess proton monolayer is also consistent with the known "electric double layer" phenomenon since the excess proton layer can be understood as an extension from the secondary (proton) layer of the anode's "electric double layer" around the proton-conductive water body surface. ${ }^{7}$ From here, we can also understand that the protons and ions of any permanent "electric double layer" formed as a result of membrane surface's fixed charges (e.g., the negatively charged phosphate groups of phospholipids) that permanently attract ions are irrelevant to the membrane potential $\Delta \psi$ in the protonic bioenergetics. ${ }^{9}$ This understanding is consistent with the conclusion made in the previous study: ${ }^{43}$ "membrane surfacefixed-charges-attracted protons are not relevant to the protonic motive force" that drives ATP synthesis.

As previously reported, ${ }^{9}$ we have recently demonstrated a protonic capacitor: the formation of a transmembrane electrostatically localized layer of excess protons at the watermembrane interface in biomimetic experiments using an anode water-membrane-water cathode system. ${ }^{19}$ The experimental demonstration of the excess anions-membrane-excess proton capacitor provided a proof-of-principle mimicking of an energized biological membrane system such as a mitochondrion in an energized resting state. ${ }^{7}$ Through an application of the Lee transmembrane electrostatic proton localization theory, the bioenergetic significance of mitochondrial cristae formation has now also been successfully elucidated. ${ }^{44}$

In this paper, the newly formulated pmf equation of refs 6, 9, and 40 is now extended to systematically calculate the energetics including the protonic motive force (pmf) and Gibbs free energy employing our experimentally measured cation-proton exchange equilibrium constants ${ }^{7}$ for sodium, potassium, and magnesium and using the existing experimental data of the alkalophilic bacterium $B$. pseudofirmus. ${ }^{2-4}$ Surprisingly, very large pmf values are found, which not only can well explain how these organisms are able synthesize $\mathrm{ATP}^{24-26}$ but also significantly exceed the maximum pmf that could be supported by the respiratory redox-driven proton pump system. Remark- 
ably, as reported below, this subsequently resulted in a major new finding that the alkalophilic bacteria are not only chemotrophs but also have a thermotrophic feature that can create significant amounts of Gibbs free energy through isothermal utilization of environmental heat energy with electrostatically localized protons (protonic thermal motion kinetic energy) to do useful work such as driving ATP synthesis. Therefore, the study reported here through the energetic analysis of a well-defined biological system, alkalophilic bacterium, represents not only a significant progress in localized proton-coupling bioenergetics but also a major discovery for a novel biophysical energy transduction phenomenon: isothermal environmental heat energy utilization by transmembrane electrostatically localized protons at the liquid-membrane interface to do useful work such as driving ATP synthesis.

\section{RESULTS AND DISCUSSION}

Effect of Surface Localized Protons on the Protonic Motive Force. The effect of surface localized protons on the protonic motive force (pmf) has now been analyzed using the newly formulated pmf equations (eqs 4-7) based on the transmembrane electrostatic proton localization theoretical framework (presented in the Materials and Methods section). Table 1 shows pmf values calculated from a set of the author's newly formulated pmf equations (eqs 5-7) under the given $B$. pseudofirmus $\mathrm{OF} 4$ culture medium $\mathrm{pH}$, cytoplasmic $\mathrm{pH}$, and transmembrane electrical potential difference conditions and taking the effect of cation exchange into account. The calculated pmf as a function of the culture medium $\mathrm{pH}$ is also displayed in Figure 1, in comparison to the classic pmf (eq 1) and local pmf (the last term in eq 5) contributions. It is apparent from these results that the surface-localized protons dominantly contribute to the overall strength of the protonic motive force.

As listed in Table 1, at the culture medium $\mathrm{pH}\left(\mathrm{pH}_{\mathrm{pB}}\right)$ of 7.5, the localized proton concentration $\left[\mathrm{H}_{\mathrm{L}}^{+}\right]$at the liquidmembrane interface is as high as $1.28 \times 10^{-2} \mathrm{M}$. As the culture

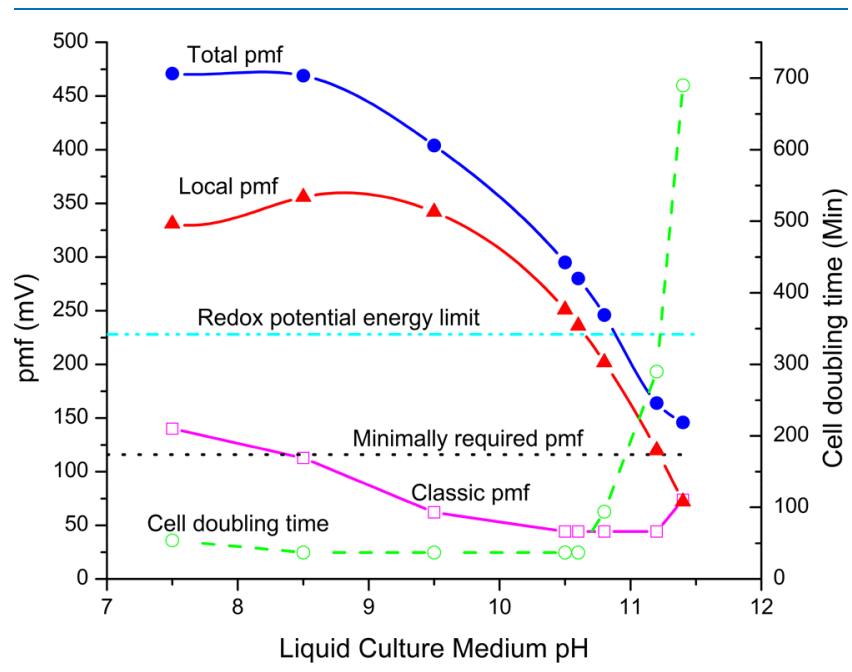

Figure 1. Calculated total, local, and classic pmf values of $B$. pseudofirmus OF4 as a function of external $\mathrm{pH}$ compared to the minimum value $(115 \mathrm{mV})$ required to synthesize ATP and to the maximum possible value (redox potential energy pmf limit: $228 \mathrm{mV}$ ) that could be supported thermodynamically by the respiratory redoxdriven proton pump system. The measured cell population growth doubling times are also superimposed. adapted with permission from ref 23. Copyright 2005 Elsevier medium $\mathrm{pH}\left(\mathrm{pH}_{\mathrm{pB}}\right)$ increased from 7.5 up to 11.4 , the localized proton concentration $\left[\mathrm{H}_{\mathrm{L}}^{+}\right]$decreased from $1.28 \times 10^{-2} \mathrm{M}$ to as low as $6.21 \times 10^{-11} \mathrm{M}$, primarily because the exchange reduction factor (the denominator in eq 7) increased from 1.50 to as big as $3.96 \times 10^{8}$ under the experimental conditions. That is, as the liquid culture medium $\mathrm{pH}\left(\mathrm{pH}_{\mathrm{pB}}\right)$ increases, the thermodynamic equilibrium for the cation-proton exchange process appears to shift for more non-proton cations such as $\mathrm{Na}^{+}, \mathrm{K}^{+}$, and $\mathrm{Mg}^{2+}$ to occupy the liquid-membrane interface, thus reducing the localized proton concentration $\left[\mathrm{H}_{\mathrm{L}}^{+}\right]$. However, even at the culture medium $\mathrm{pH}\left(\mathrm{pH}_{\mathrm{pB}}\right)$ of 11.4 , the localized proton concentration $\left(6.21 \times 10^{-11} \mathrm{M}\right)$ is still an order of magnitude higher than the culture medium bulk-phase proton concentration $\left[\mathrm{H}_{\mathrm{pB}}^{+}\right]$of $3.98 \times 10^{-12} \mathrm{M}$.

Use of the localized proton concentration $\left[\mathrm{H}_{\mathrm{L}}^{+}\right]$and the medium bulk-phase proton concentration $\left[\mathrm{H}_{\mathrm{pB}}^{+}\right]$can now readily calculate the local pmf according to the third term of the author's newly formulated protonic motive force (pmf) equation (eq 5). The values of the calculated local pmf are listed in Table 1 and plotted in Figure 1. As shown in Figure 1, the local pmf is typically much bigger than the classic pmf as calculated from the textbook Mitchellian pmf equation (eq 1). Therefore, the textbook Mitchellian pmf equation missed a major pmf component here.

The total pmf values, which equals to the sum of the local pmf and the classic pmf, are also listed in Table 1 and presented in Figure 1. As shown in Figure 1, the overall pattern of the total pmf amazingly matched with the B. pseudofirmus OF4 cell population growth observed experimentally with the cell population growth doubling times. That is, the pattern of the total pmf calculated through the newly formulated pmf equations (eqs 4-7) from the ground up using the real experimental data including the liquid culture medium $\mathrm{pH}$ $\left(\mathrm{pH}_{\mathrm{pB}}\right)$ and cation concentrations, the measured membrane potential $(\Delta \psi)$ and cytoplasmic $\mathrm{pH}\left(\mathrm{pH}_{\mathrm{nB}}\right)$ excellently matched with the pattern of the observed cell population growth doubling times. This indicates that we really are on something real.

A pmf of at least $115 \mathrm{mV}$ (equivalent to a Gibbs free energy of $-11.1 \mathrm{~kJ} / \mathrm{mol}$ ) is needed to overcome the known phosphorylation potential $(-497 \mathrm{mV}$ at culture medium $\mathrm{pH} 10.5)$ for ATP synthesis ${ }^{21}$ with a proton-to-ATP ratio of $13 / 3(497 \mathrm{mV} /$ $4.33=115 \mathrm{mV}$ ). The proton-to-ATP ratio of $13 / 3$ is according to the known structures and functions of the B. pseudofirmus $\mathrm{F}_{0} \mathrm{~F}_{1}$-ATP synthase, which has three catalytic sites for ATP synthesis, driven by a flow of 13 protons per revolution through the $13 \mathrm{c}$-subunits in its nanometer-scale molecular turbine ring. $^{45,46}$

As shown in Figure 1, the classic pmf such as $44 \mathrm{mV}$ at liquid culture medium $\mathrm{pH} 10.5$ is far below the minimally required $\mathrm{pmf}$ of $115 \mathrm{mV}$ to drive ATP synthesis. Also, the pattern of the classic pmf does not match or make any sense with the observed cell population growth doubling times. For example, when the classic pmf goes up from $44 \mathrm{mV}$ (at liquid culture medium $\mathrm{pH}$ 11.2 ) to $74 \mathrm{mV}$ (at $\mathrm{pH} 11.4$ ), one would expect that the cell population growth doubling times should go down because the increased pmf would support cell growth. However, the observed cell population growth doubling time went up (growth slower) instead of down. Neither the classic pmf value nor its pattern could explain the observed cell growth. This explains why the textbook Mitchellian pmf equation (eq 1) could never explain this bioenergetic problem, which was almost like an elephant in a room for the scientists who understood the issue 
here over the last three decades in regard to how these microbes are able to synthesize ATP. ${ }^{24-26}$

On the other hand, the total pmf values including the contributions from the localized protons as shown in Figure 1 are well above the minimally required value of $115 \mathrm{mV}$ in driving the synthesis of ATP, although the classic Mitchellian pmf is below this minimally required $\mathrm{pmf}$ for $\mathrm{pH}$ values at and above 8.5. This result can well explain why the B. pseudofirmus OF4 culture keeps such an excellent growth rate (cell doubling times less than $100 \mathrm{~min}$ ) with culture $\mathrm{pH}$ ranging from 7.5 to 10.8 . Furthermore, the decrease in total pmf when the liquid culture $\mathrm{pH}$ is raised beyond 10.8 , owing to decreasing contributions from the localized protons caused by cation exchange, correlates amazingly well with the rapid increase in growth cell doubling times for $\mathrm{pH}$ values above 10.6. Particularly, the downward curve pattern of the newly calculated total pmf (as shown in Figure 1) matches excellently with the upward curve pattern of the experimentally measured cell doubling time in the entire range of the liquid cell culture medium $\mathrm{pH}$ from 7.5 to 11.4. Therefore, evidently, this result (data shown in Table 1 and Figure 1) from the use of the newly developed pmf expression in eqs 5-7 has now, for the first time, consistently provided an excellent elucidation for the 30 year longstanding energetic conundrum $^{24}$ in alkalophilic bacteria in regard to how they are able to synthesize ATP and grow.

Localized Protons Utilize Environmental Heat Energy to Synthesize ATP. It is encouraging that the total pmf values calculated by eq 5 for B. pseudofirmus OF4 are all larger than the minimum value required to synthesize ATP. However, it is a surprising result that several of these values (such as 471, 469, 404 , and $295 \mathrm{mV}$ ) are significantly larger than the maximum pmf $(228 \mathrm{mV}$, equivalent to a Gibbs free energy of $-22.0 \mathrm{~kJ} / \mathrm{mol}$ ) that could be supported thermodynamically by the respiratory redox-driven proton pump system. This is also shown in Figure 1.

The maximum possible pmf value that could be supported by the respiratory redox-driven proton pump system, such as the one in B. pseudofirmus OF4, is only about $228 \mathrm{mV}$. The redox potential difference between the electron donor $\mathrm{NADH}\left(E_{\mathrm{m}, 7}=\right.$ $-320 \mathrm{mV})$ to the terminal electron acceptor $\mathrm{O}_{2}\left(E_{\mathrm{m}, 7}=+820\right.$ $\mathrm{mV})$ in this system is known to be about $1140 \mathrm{mV} .^{16}$ For each pair of electrons from $\mathrm{NADH}$ to pass through the respiratory chain to the terminal electron acceptor $\mathrm{O}_{2}$, the system drives the translocation of 10 protons across the membrane from the cytoplasm to the culture medium outside the cell. That is, it couples the translocation of 5 protons per electron across the membrane. Therefore, the maximum pmf that could be generated is about $228 \mathrm{mV}$ per proton ( $1140 \mathrm{mV} / 5$ protons).

How is it possible that the total transmembrane pmf exceeds the limit imposed by the respiratory redox-driven proton pump system? We now understand here that the local pmf from localized protons is not constrained by the overall energetics limit of the redox-driven proton pump system and that the ambient environmental heat (representing the dissipated form of heat energy) in the cell culture medium is effectively utilized by the localized protons for ATP synthesis (driving the synthesis of ATP from ADP and Pi through $\mathrm{F}_{0} \mathrm{~F}_{1}$-ATP synthase), for the following reasons.

First, the localized protons are not free to move away from the membrane-liquid interface; they are electrostatically held at the membrane surface. ${ }^{19,20}$ Consequently, their thermal motion (environmental heat) will cause some to enter the protonic opening ( $F_{0}$ proton channel inlet) of the ATP synthase and be used to drive its nanometer-scale molecular turbine for synthesis of ATP from ADP and $\mathrm{Pi}^{45,47,48}$

Secondly, the localized protons may not be directly coupled to the membrane-embedded redox proton pumps: namely, the respiratory chain complexes I, III, and IV. ${ }^{49}$ If they were, their ability to do work would be constrained by the energetics of the respiratory redox-driven proton pump system. A natural explanation of why this does not occur would be that the exit points for the translocated protons are outside of the surface layer of the electrostatically localized protons. Furthermore, to effectively make use of the localized proton thermal motion, the protonic entry point for the ATP synthase should be inside the localized proton surface layer. In this way, the redox-driven proton pump activity interacts with the proton activity in the bulk liquid phases but not with that of the localized proton layer at the liquid-membrane interface. Only the transmembrane electrical potential difference and the bulk-phase proton activity at the two sides of the membrane equilibrate with the protonpumping respiratory electron-transport-chain activity. Consequently, the localized proton thermal motion provides additional free energy that may be utilized isothermally by the ATP synthase and is not limited by $228 \mathrm{mV}$.

The structures of bacterial respiratory membrane protein complexes, such as those in B. pseudofirmus OF4, are not well known. However, they are believed to be similar to the redoxdriven proton-pumping complexes in mitochondria, which have been more extensively studied. Quite remarkably, the known structures of the mitochondrial respiratory protein complexes, as determined by cryo-electron microscopy and other molecular structural studies, ${ }^{49-57}$ fit amazingly well with this explanation. As shown in Figure 2, every one of the mitochondrial respiratory

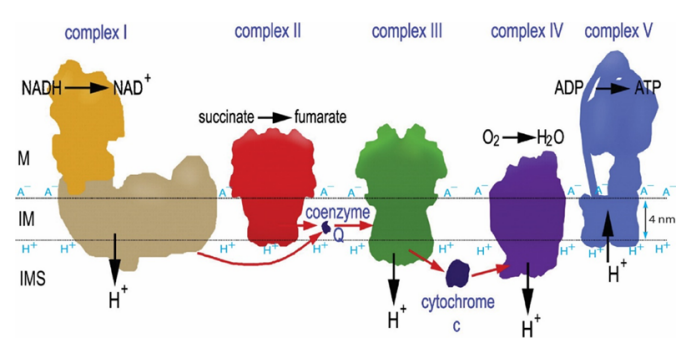

Figure 2. Known structures of mitochondrial respiratory membrane protein complexes I, II, III, IV, and ATP synthase (complex V) in relation to the location of the cell membrane surfaces indicated by the horizontal dotted lines. The thickness of the membrane lipid bilayer (in between two dotted lines) is known to be about $4 \mathrm{~nm}$, with which as reference, the protonic outlets of the proton pumping complexes I, III, and IV are seen to be all protruded by about 1 to $3 \mathrm{~nm}$ into the bulk liquid phase while the protonic inlet of ATP synthase (complex V) is located at the localized proton layer along the membrane surface. Adapted and modified with permission from ref 50. Copyright 2009 Elsevier.

proton-pumping protein complexes I, III, and IV protonic outlets is indeed protruded away from the membrane surface by about 1 to $3 \mathrm{~nm}$ into the bulk liquid phase, while the protonic inlet of the ATP synthase (complex V) is located right at the localized proton layer along the membrane surface. Furthermore, complex II, which does not pump protons and thus does not need to be protruded, is indeed not protruded away from the membrane surface at the intermembrane space (IMS) side as the author also predicted. Therefore, the discovery of isothermal environmental heat energy utilization by electrostatically localized protons at liquid-membrane interface is also well 

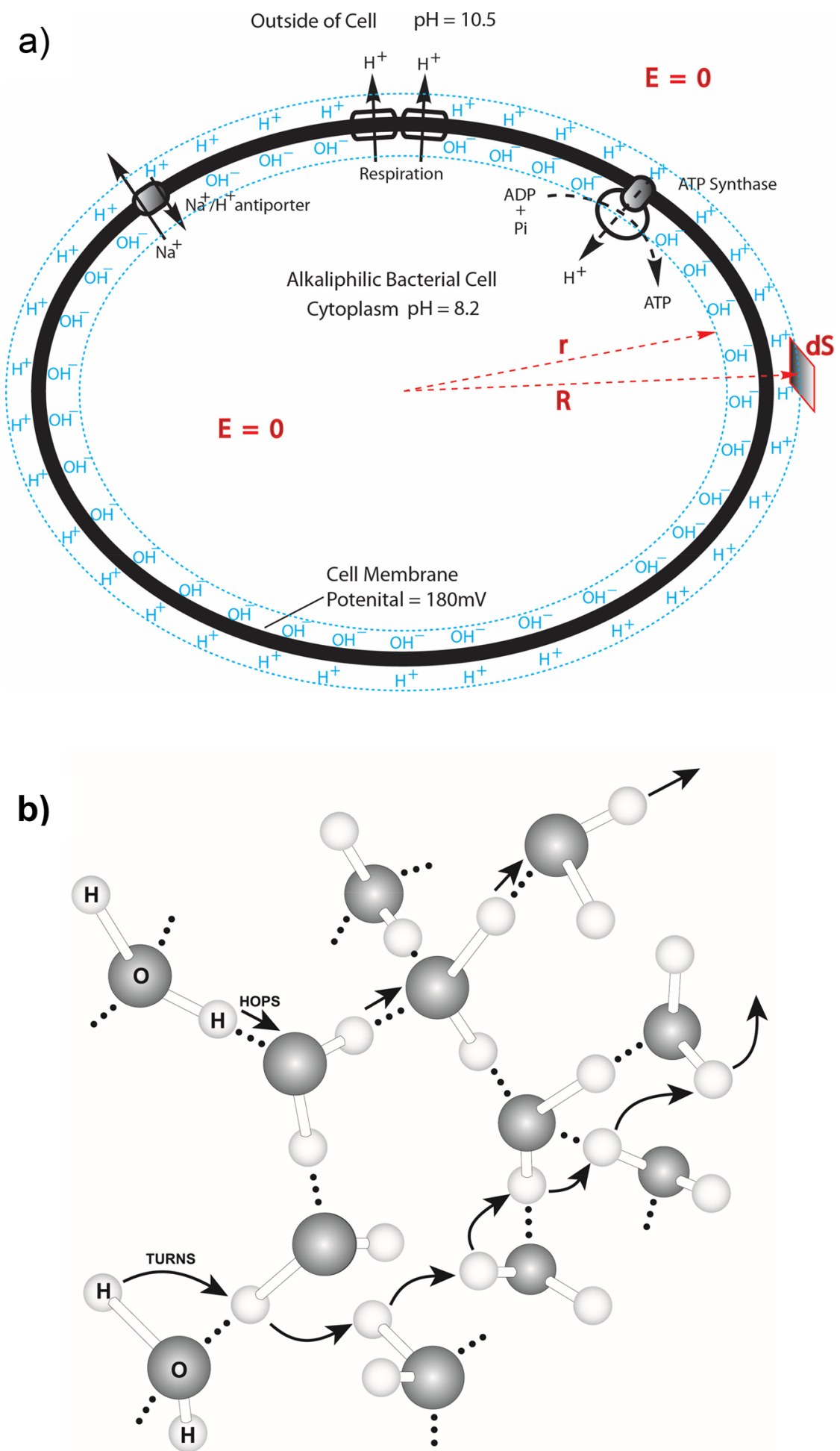

Figure 3. (a) Proton-electrostatic localization model shown as "a proton capacitor-like structure" illustrating how excess protons $\left(\mathrm{H}^{+}\right)$and hydroxyl ions $\left(\mathrm{OH}^{-}\right)$are transmembrane electrostatically localized at the water-membrane interfaces along the two sides of a bacterial cell membrane before proton-cation exchange as it would be in an idealized pure water-membrane-water system; (b) protons can quickly translocate among water molecules by the "hops and turns" mechanism. adapted with permission from ref 5. Copyright 2012 Creative Commons.

corroborated with the known structures of the ATP synthase (complex V) and complexes I, II, III, and IV in the asymmetric biological membrane.

Apparently, the billion years of natural evolution already made the biological membrane asymmetric structures (Figure 2) perfect to constitute this amazing isothermal environmental heat utilization function with localized protons. This is also remarkably corroborated with our experimental observations that the localized protons at a liquid-membrane interface are "not detectable to a $\mathrm{pH}$ electrode in the bulk liquid phase". 19,20,58 The biological systems apparently take advantage of this feature by "purposely" protruding the protonic outlets of their respiratory proton-pumping protein complexes I, III and IV into the bulk liquid phase to avoid the contact with localized 
protons at the liquid-membrane interface while keeping the protonic mouth of the ATP synthase precisely at the membrane surface to effectively utilize the localized protons that dominantly contribute to the total protonic motive force as shown by the data in Figure 1.

Therefore, we can now start to understand that the transmembrane electrostatically localized protons in combination with the asymmetric structural features of biological membrane especially in regard to the positions of the protonic pump outlets and the mouth of the localized proton users such as ATP synthase with respect to the localized proton layer along the $p$ side of the membrane may constitute this special isothermal environmental heat energy utilization function. This is consistent with the understanding that the molecular thermal motion energy utilized isothermally as local pmf (equivalent to Gibbs free energy) is from the environment heat associated with the electrostatically localized protons. No new energy is created nor destroyed here. Therefore, this special isothermal environmental heat energy utilization function perfectly follows the first law (conservation of mass and energy) of thermodynamics but is not necessarily constrained by the redox-driven proton pump system that apparently well follows the second law of thermodynamics. Fundamentally, it is the transmembrane electrostatic proton localization with the effect of water as a protonic conductor (Figure 3) that enables the formation of a localized excess proton layer at the liquidmembrane interface over the mouths of the pmf users including the $\mathrm{F}_{0} \mathrm{~F}_{1}$-ATP synthase (Figure 2). The formation of a localized excess proton layer at the water-membrane interface apparently results in some kind of "negative entropy effect" $20,39,59,60$ that brings the excess protons to the mouths of the pmf users where the protons can isothermally utilize their molecular thermal motions energy (proton thermal kinetic energy $k_{\mathrm{B}} T$ ) possibly including their Brownian motion to push through the doors of $\mathrm{F}_{0} \mathrm{~F}_{1}$-ATP synthase in driving ATP synthesis. Note, in the third term of eq 5 for the local pmf, the utilization of proton thermal kinetic energy $k_{\mathrm{B}} T$ is expressed as $R T\left(=k_{\mathrm{B}} T \cdot N_{\mathrm{A}}\right)$, which equals to the product of the Boltzmann constant $k_{\mathrm{B}}$, the temperature $T$, and the Avogadro constant $N_{\mathrm{A}}$.

For the delocalized protons in the bulk liquid phase that are far away from the membrane surface, their thermal motions are not within the striking distance for them to hit into the $\mathrm{F}_{0} \mathrm{~F}_{1}$ ATP synthase protonic channel to drive the rotary molecular machinery for ATP synthesis. Theoretically, to some extent, the delocalized protons could also do the work when they are at the liquid-membrane interface near the $\mathrm{F}_{0} \mathrm{~F}_{1}$-ATP synthase. Therefore, the thermal energy factor $R T\left(=k_{\mathrm{B}} T \cdot N_{\mathrm{A}}\right)$ is also in the second term of the newly formulated pmf equation (eq 5 ). However, the value of $\log _{10}\left(\left[\mathrm{H}_{\mathrm{pB}}^{+}\right] /\left[\mathrm{H}_{\mathrm{nB}}^{+}\right]\right)$was nearly zero or negative in this case; consequently, the delocalized protons did not significantly contribute to the isothermal utilization of environmental heat in driving ATP synthesis here.

It is worth noting that the isothermal utilization of environmental heat (dissipated-heat energy) by transmembrane electrostatically localized protons at the liquid-membrane interface occurs without any physical phase transition such as the conventional liquid-gas phase change that would be required in the classic latent heat of water vaporization. Both the liquid phase inside the cell and the liquid culture medium outside the cell stay the same during the isothermal utilization of environmental heat through electrostatically localized protons. Furthermore, there is no temperature difference across the biological membrane. Therefore, the utilization of dissipated- heat energy from the ambient temperature environment through transmembrane electrostatically localized protons at the liquidmembrane interface in driving ATP synthesis reported here is indeed a novel isothermal energy renewal process without involving any phase transition.

Negative Gibbs Free Energy Change through Protonic Isothermal Environmental Heat Energy Utilization. Note, the protonic motive force is equivalent to Gibbs free energy $\Delta G$ according to a simple relation with the Faraday constant $(F)$ as in the following equation:

$$
\Delta G=-F \times \mathrm{pmf}
$$

Consequently, the amount of local pmf Gibbs free energy $\left(\Delta G_{\mathrm{L}}\right)$ resulting from this special isothermal environmental heat energy utilization function with localized protons can be calculated using the following equation:

$$
\Delta G_{\mathrm{L}}=-2.3 R T \log _{10}\left(1+\left[\mathrm{H}_{\mathrm{L}}^{+}\right] /\left[\mathrm{H}_{\mathrm{pB}}^{+}\right]\right)
$$

According to this equation (eq 3), as long as the localized proton concentration $\left[\mathrm{H}_{\mathrm{L}}^{+}\right]$is above zero, the Gibbs free energy $\left(\Delta G_{\mathrm{L}}\right)$ change is indeed a negative number. That is, the Gibbs free energy $\left(\Delta G_{\mathrm{L}}\right)$ change for the isothermal environmental heat utilization process is indeed negative if there is a localized proton concentration $\left[\mathrm{H}_{\mathrm{L}}^{+}\right]$(above zero). The amount of Gibbs free energy $\Delta G_{\mathrm{L}}$ created from the utilization of environmental heat through the localized protons is a function of the localized proton concentration $\left[\mathrm{H}_{\mathrm{L}}^{+}\right]$as shown in eq 3 .

The proton-coupling bioenergetics systems widely operate in nearly all organisms known today. Therefore, through the present study as reported above, it is now quite clear that this special biological isothermal environmental heat utilization process associated with electrostatically localized protons has occurred probably for billions of years already on Earth. As shown in Figure 1, the amount of local pmf as calculated according to the third term of the newly formulated pmf equation (eq 5) quantitatively represents the activity of this amazing isothermal environmental heat (proton thermal kinetic energy $k_{\mathrm{B}} T$ ) utilization function with localized protons. Accordingly, it is the ratio $\left(\left[\mathrm{H}_{\mathrm{L}}^{+}\right] /\left[\mathrm{H}_{\mathrm{pB}}^{+}\right]\right)$of the localized proton concentration $\left[\mathrm{H}_{\mathrm{L}}^{+}\right]$at the membrane-liquid interface to the bulk-phase proton concentration $\left[\mathrm{H}_{\mathrm{pB}}^{+}\right]$at the same $\mathrm{p}$ side of the membrane that mathematically relates to this special isothermal environmental heat (protonic thermal kinetic energy $k_{\mathrm{B}} T$ ) utilization function, which is quite surprising.

The local pmf represents a very large portion of the total pmf. For example, when the liquid culture medium $\mathrm{pH}$ was 7.5 , the local pmf $(331 \mathrm{mV}$, equivalent to a Gibbs free energy of -32.0 $\mathrm{kJ} / \mathrm{mol}$ ) represents about $70 \%$ of the total pmf $(471 \mathrm{mV}$, equivalent to a Gibbs free energy of $-45.5 \mathrm{~kJ} / \mathrm{mol}$ ) as listed in Table 1 . When the liquid culture medium $\mathrm{pH}$ was raised to 10.5 , the local pmf $(251 \mathrm{mV}$, equivalent to a Gibbs free energy of $-24.2 \mathrm{~kJ} / \mathrm{mol})$ represents $85 \%$ of the total pmf $(295 \mathrm{mV}$, equivalent to a Gibbs free energy of $-28.5 \mathrm{~kJ} / \mathrm{mol}$ ). Since $\mathrm{pmf}$ is equivalent to Gibbs free energy $\Delta G$ according to eq 2 , this result (data in Table 1) also showed that the values of Gibbs free energy $(\Delta G)$ created through isothermal utilization of environmental heat thermal energy by the localized protons are much more than the classic pmf free energy. That is, the bacteria obtain as much as $70-85 \%$ of the total pmf Gibbs free energy from the isothermal utilization of environmental heat energy associated with the localized protons. Therefore, it is now quite clear that 
the bacteria have a significant thermotrophic property ${ }^{61}$ in addition to being chemotrophs.

In the past centuries, it was thought to be completely impossible to isothermally utilize the environmental heat "dead energy" dissipated in the ambient environment to do any useful work, which is also known as one of the classic second law statements. ${ }^{1,62,63}$ That classic belief is still largely true for many systems and processes around us including the cars and computers that we use. However, we now know, in certain special biophysical molecular systems such as the transmembrane electrostatically localized protons at the liquidmembrane interface, that classic belief may not necessarily be always true.

That is, the second law remains still as a very good law. However, it does not necessarily have to be always universal as implicated also by a number of independent studies. ${ }^{20,61,63-73}$ For example, many of the biological processes such as glycolysis, tricarboxylic acid cycle, and redox-driven proton transport apparently well follow the second law. Meanwhile, we now also understand, the special protonic isothermal environmental heat energy utilization function as reported here perfectly follows the first law (conservation of mass and energy) of thermodynamics but is not necessarily constrained by the redox-driven proton pump system that apparently well follows the second law of thermodynamics.

When the localized protons utilize environmental heat $\left(k_{\mathrm{B}} T\right)$ in helping drive the synthesis of ATP from ADP and Pi through the $\mathrm{F}_{0} \mathrm{~F}_{1}$-ATP synthase (Figure 2 ), a fraction of the environmental heat $\left(k_{\mathrm{B}} T\right)$ energy may be locked into the chemical form of energy in ATP molecules; and it thus would result in a small drop in the environmental temperature theoretically because of the localized proton-associated isothermal environmental heat utilization. However, in the cell, there are many other processes (including the glycolysis, tricarboxylic acid cycle, and the redoxdriven proton-pumping electron transport activities as well as the ATP utilization processes such as ATP hydrolysis) releasing heat energy, which could mask the isothermal environmental heat utilization process. That is, the energetic phenomenon of the cell may represent a complicated mixture of the chemotrophic processes and the isothermal environmental heat energy utilization process. This could probably also explain why it took so long for human beings on Earth to finally figure this out here.

Therefore, the discovery reported here may have profound scientific and practical implications in bettering the fundamental understanding of bioenergetics and energy renewal ${ }^{20}$ for sustainable development on Earth. For example, with the new knowledge learned from this discovery, it may be possible to have the benefits of mimicking this biophysical molecular scale process to create a new way ${ }^{20,39,71}$ in producing useful energy by isothermally utilizing the environmental heat energy from the ambient environment.

\section{CONCLUSIONS WITH THE DISCOVERY}

Taking transmembrane electrostatically localized protons into account with the newly developed expression of eqs 5-7 provides a large enough pmf to synthesize ATP in the $B$. pseudofirmus OF4 cells. Furthermore, owing to the reduction in localized protons due to increasing cation exchanges, the pmf decreases with increasing culture medium $\mathrm{pH}$, well consistent with the observed decrease in bacteria growth rates. None of these features could be explained with the textbook Mitchellian pmf equation. ${ }^{10,74,75}$ It is remarkably shown here, for the first time, that the curve pattern of the newly calculated total pmf excellently matches with that of the experimentally measured cell doubling time, which now clearly elucidates the decadeslongstanding energetic conundrum ${ }^{24-26}$ as to how the alkalophilic bacteria are able to synthesize ATP and grow.

More importantly, we now understand with well-corroborated scientific evidence that biological membrane systems can isothermally utilize low-grade environmental heat energy associated with the ambient temperature thermal motion of transmembrane electrostatically localized protons (proton thermal kinetic energy $k_{\mathrm{B}} T$ ) to perform useful work driving the synthesis of ATP. This amazing phenomenon can occur where the outlets of the redox-driven proton pumps are protruded away from the membrane surface to deliver protons into the bulk liquid phase, while the protonic inlet of the ATP synthase is located at the membrane surface. These features as predicted also match excellently with the true structures of mitochondrial respiratory membrane protein complexes I, II, III, and IV, and ATP synthase in relation to the location of the biological membrane surfaces. ${ }^{49-57}$

The Gibbs free energy $\left(\Delta G_{\mathrm{L}}\right)$ change for the isothermal environmental heat utilization process is indeed negative as long as the localized proton concentration $\left[\mathrm{H}_{\mathrm{L}}^{+}\right]$is above zero. The amount of Gibbs free energy $\Delta G_{L}$ created from the utilization of environmental heat through the localized protons is a function of the localized proton concentration $\left[\mathrm{H}_{\mathrm{L}}^{+}\right]$as shown in eq 3 . It is the ratio $\left(\left[\mathrm{H}_{\mathrm{L}}^{+}\right] /\left[\mathrm{H}_{\mathrm{pB}}^{+}\right]\right)$of the localized proton concentration $\left[\mathrm{H}_{\mathrm{L}}^{+}\right]$at the membrane-liquid interface to the bulk-phase proton concentration $\left[\mathrm{H}_{\mathrm{pB}}^{+}\right]$at the same $\mathrm{p}$ side of the membrane that mathematically relates to this special isothermal environmental heat (protonic thermal kinetic energy $k_{\mathrm{B}} T$ ) utilization function.

Surprisingly, the local pmf represents a very large portion of the total pmf. That is, the bacteria obtain as much as $70-85 \%$ of the total pmf Gibbs free energy from the isothermal utilization of environmental heat energy associated with the localized protons. Consequently, the bacteria have a significant thermotrophic feature $^{61}$ in addition to being chemotrophs.

Therefore, it is now quite clear that the pmf (equivalent to Gibbs free energy) from the isothermal utilization of environmental heat energy through the transmembrane electrostatically localized protons is not constrained by the overall energetics of the redox-driven proton pump system because of the following: (a) as a protonic capacitor feature, the localized protons are not free to move away from the membrane surface; (b) as an asymmetric property of the biological membrane, the proton pumps embedded in the cell membrane extend beyond the localized proton layer; and (c) as another natural property of the asymmetric biological membrane, the protonic inlet mouth of the ATP synthase that accepts protons is located within this localized proton layer. The biological systems can isothermally utilize the environmental heat energy through electrostatically localized protons to do useful work such as driving the synthesis of ATP. This is a major discovery that may have profound scientific implications in bettering the fundamental understanding of bioenergetics and isothermal environmental heat energy utilization in biological systems.

\section{MATERIALS AND METHODS WITH EQUATIONS}

Theoretical Framework for Protons Localized at Cell Membrane Surfaces. The basic process that leads to synthesis of ATP in cells and hence cell growth involves creating an excess number protons on one side of a biological membrane accompanied by a corresponding number of hydroxyl ions on 
the other side, for instance, through the respiratory electrontransport-coupled proton pumps across the membrane. Subsequently, protons are transported to the protonic inlet of the ATP synthase complex embedded in the cell membrane.

Based on the transmembrane electrostatic proton localization theory, 5,6,8,9,39 excess positively charged protons in an aqueous medium will electrostatically repel each other to become localized at the membrane surface on one side of a cell membrane, attracting an equal number of excess negatively charged hydroxyl anions to the other side of the membrane to form a "protonic capacitor" (Figure 3a). This theory is built on the premise that a liquid water body can act as a protonic conductor since protons can quickly transfer among water molecules by the "hops and turns" mechanism (Figure 3b) first outlined by Grotthuss. ${ }^{76-78}$

As illustrated in Figure $3 \mathrm{a}$ and reported with great details in refs 6,9 , the theoretical support of the Lee transmembrane proton electrostatic localization model ${ }^{5,6,8,39}$ with a protonic capacitor system was first provided mathematically using the Gauss law integration equation of electrostatics with a differential surface element $\mathbf{d} \mathbf{S}$ in polar coordinates $\mathbf{r}$ and $\mathbf{R}$ based on the premise of water as a protonic conductor and the fact that there can be zero electric field $\mathbf{E}$ inside a conductor. Indeed, such a protonic capacitor system with localized protons at a liquid-membrane interface has recently been verified experimentally in the Lee laboratory using a biomimetic watermembrane-water electrolysis system setup. ${ }^{7,19,20,39,58}$

Therefore, a generalized equation for the protonic motive force (pmf) across a biological membrane taking into account the surface localized protons was introduced in refs 6, 9, 39 as

$$
\mathrm{pmf}=\Delta \psi+\frac{2.3 R T}{F} \log _{10}\left(\left\{\left[\mathrm{H}_{\mathrm{L}}^{+}\right]+\left[\mathrm{H}_{\mathrm{pB}}^{+}\right]\right\} /\left[\mathrm{H}_{\mathrm{nB}}^{+}\right]\right)
$$

Here, $\left[\mathrm{H}_{\mathrm{L}}^{+}\right]$is the concentration of the surface-localized protons, $\left[\mathrm{H}_{\mathrm{pB}}^{+}\right]$is the proton concentration in the periplasmic bulk aqueous phase, and $\left[\mathrm{H}_{\mathrm{nB}}^{+}\right]$is the proton concentration in the cytoplasmic bulk phase. Through algebraic rearrangement, this equation is now rewritten to isolate the contribution of the localized protons as follows:

$$
\begin{aligned}
\mathrm{pmf}= & \Delta \psi+\frac{2.3 R T}{F} \log _{10}\left(\left[\mathrm{H}_{\mathrm{pB}}^{+}\right] /\left[\mathrm{H}_{\mathrm{nB}}^{+}\right]\right)+\frac{2.3 R T}{F} \\
& \log _{10}\left(1+\left[\mathrm{H}_{\mathrm{L}}^{+}\right] /\left[\mathrm{H}_{\mathrm{pB}}^{+}\right]\right)
\end{aligned}
$$

The first two terms of eq 5 comprise the Mitchellian "classic" bulk phase-to-bulk phase pmf, as noted in eq 1, whereas the last term accounts for the "local" pmf from the transmembrane electrostatically localized protons.

For an idealized protonic capacitor, the concentration of the ideal localized protons $\left[\mathrm{H}_{\mathrm{L}}^{+}\right]^{0}$ at the membrane-water interface is related to the transmembrane electrical potential difference $\Delta \psi$ by

$$
\left[\mathrm{H}_{\mathrm{L}}^{+}\right]^{0}=\frac{C}{S} \cdot \frac{\Delta \psi}{l \cdot F}=\frac{\Delta \psi \cdot \kappa \cdot \varepsilon_{\mathrm{o}}}{d \cdot l \cdot F}
$$

where $C / S$ is the specific membrane capacitance per unit surface area, $l$ is the thickness of the localized proton layer, $\kappa$ is the dielectric constant of the membrane, $\varepsilon_{\mathrm{o}}$ is the electric permittivity, and $d$ is the membrane thickness. In actual biological systems, non-proton cations in the aqueous media may exchange with protons localized at the membrane surface and thereby reduce their concentration. To account for this effect, we use

$$
\left[\mathrm{H}_{\mathrm{L}}^{+}\right]=\frac{\left[\mathrm{H}_{\mathrm{L}}^{+}\right]^{0}}{\prod_{i=1}^{n}\left\{K_{P i}\left(\frac{\left[\mathrm{M}_{\mathrm{pB}}^{i+}\right]}{\left[\mathrm{H}_{\mathrm{pB}}^{+}\right]}\right)+1\right\}}
$$

for the resulting concentration of localized surface protons. Here, $\left[\mathrm{M}_{\mathrm{pB}}^{i+}\right]$ is the concentration of a given non-proton cation, and $K_{P i}$ is the equilibrium constant for the cation to exchange with the localized protons.

It is noteworthy that all the physical quantities appearing in eqs 4-7 may, in principle, be determined experimentally with no freely adjustable parameters. For the membrane and proton layer parameters in eq 6, the calculations reported here have taken $C / S=13.2 \mathrm{mF} / \mathrm{m}^{2}$ as an averaged membrane capacitance based on measured experimental data ${ }^{79}$ and $l=1 \mathrm{~nm}$, which, as discussed in refs $6,9,19$, is a reasonable thickness of the localized proton layer. Also, as discussed above, the localized proton layer thickness (l) may put constraints on the redox electrontransport-coupled proton pumps and proton gradient user structures that are embedded within a biological membrane.

In some of the literature, the membrane potential might somehow be reported as a "negative" number ${ }^{21-23}$ because of using an opposite reference orientation (from the cytoplasmic $n$ side to the periplasmic $\mathrm{p}$ side $)^{80,81}$ in contrast to that of the $\Delta \psi$ defined in eqs 5 and 6 from the periplasmic $p$ side to the cytoplasmic $\mathrm{n}$ side. In that case, special care should be taken to correct (remove) the negative sign for such membrane potential data reported with the opposite orientation such as that from ref 23 before applying it to the newly formulated pmf equation (eq 5) here.

In this study, the bioenergetics analysis of refs 6, 9 was extended by making a more complete treatment of the cation exchange using newly measured proton-cation exchange equilibrium constants for sodium and potassium cations ${ }^{7,58}$ to calculate the pmf and Gibbs free energy for the whole range of measured values for $\Delta \psi,\left[\mathrm{H}_{\mathrm{pB}}^{+}\right]$, and $\left[\mathrm{H}_{\mathrm{nB}}^{+}\right]$, covering $\mathrm{pH}_{\mathrm{pB}}$ values from 7.5 to 11.5 , for the well-documented B. pseudofirmus OF4 liquid culture experiments. ${ }^{21-23}$ These experimentally determined values are also shown in Figure 1. Also shown are the corresponding measured cell population growth doubling times, which were typically less than $100 \mathrm{~min}$ (excellent growth) in a culture medium $\mathrm{pH}\left(\mathrm{pH}_{\mathrm{pB}}\right)$ range from 7.5 to 10.8, except for its rapid increase (slower growth) for $\mathrm{pH}_{\mathrm{pB}}$ values between 10.8 and 11.5 (Figure 1). This work, as reported above, finally led to the discovery with well-corroborated evidence on an exciting new biological energy phenomenon: isothermal environmental heat energy utilization by transmembrane electrostatically localized protons at the liquid-membrane interface to do useful work such as driving ATP synthesis at the biophysical molecular scale.

Effect of Cation Exchange with Transmembrane Electrostatically Localized Protons. Concentrations of the cations $\mathrm{Na}^{+}, \mathrm{K}^{+}, \mathrm{Mg}^{2+}, \mathrm{Ca}^{2+}, \mathrm{NH}_{4}^{+}, \mathrm{Zn}^{2+}, \mathrm{Fe}^{2+}, \mathrm{Mn}^{2+}, \mathrm{Cu}^{2+}$, and $\mathrm{Co}^{2+}$ in the liquid culture of B. pseudofirmus OF4 are presented in Table 2. They were calculated from the composition of the culture medium reported in refs $21,82,83$. Also shown in Table 2 are the cation-proton exchange equilibrium constants $K_{P i}$ used in this study. The $K_{P i}$ values for $\mathrm{Na}^{+}$and $\mathrm{K}^{+}$were experimentally determined to be $5.07 \times 10^{-8}$ and $6.93 \times 10^{-8}$, respectively, through a study ${ }^{7,58}$ similar to that reported in ref 19 . The average of these two values $\left(6.00 \times 10^{-8}\right)$ was used to estimate $K_{P i}$ for the other monovalent cation $\mathrm{NH}_{4}{ }^{+}$. The $K_{P i}$ value of $2.1 \times 10^{-7}$ for $\mathrm{Mg}^{2+}$ was calculated from experimental 
Table 2. Experimental Cation Concentrations in B. pseudofirmus OF4 Medium, Estimated Cation-Proton Exchange Equilibrium Constants, and Calculated Cation Exchange Reduction Factors (the Denominator in Eq 7) of the Transmembrane Electrostatically Localized Proton Concentration at $\mathrm{pH}_{\mathrm{pB}}=\mathbf{1 0 . 5}$

\begin{tabular}{lllc}
$\begin{array}{c}\text { cation } \\
\text { species } \\
\mathrm{M}_{\mathrm{pB}}^{i+}\end{array}$ & $\begin{array}{c}\text { cation species } \\
\text { concentration } \\
{\left[\mathrm{M}_{\mathrm{pB}}^{i+}\right]}\end{array}$ & \multicolumn{1}{c}{$\begin{array}{c}\text { exchange equilibrium } \\
\text { constant } K_{P i}\end{array}$} & $K_{P_{i}}\left(\frac{\left[\mathrm{M}_{\mathrm{pB}}^{i+}\right]}{\left[\mathrm{H}_{\mathrm{pB}}^{+}\right]}\right)+1$ \\
$\mathrm{Na}^{+}$ & $300 \mathrm{mM}$ & $5.07 \times 10^{-8}$ & 482 \\
$\mathrm{~K}^{+}$ & $3.584 \mathrm{mM}$ & $6.93 \times 10^{-8}$ & 8.85 \\
$\mathrm{Mg}^{2+}$ & $0.1 \mathrm{mM}$ & $2.10 \times 10^{-7}$ & 1.66 \\
$\mathrm{Ca}^{2+}$ & $0.4557 \mathrm{mM}$ & $2.10 \times 10^{-7}$ & 4.03 \\
$\mathrm{NH}_{4}^{+}$ & $0.971 \mu \mathrm{M}$ & $6.00 \times 10^{-8}$ & 1.00 \\
$\mathrm{Zn}^{2+}$ & $38.08 \mu \mathrm{M}$ & $2.10 \times 10^{-7}$ & 1.25 \\
$\mathrm{Fe}^{2+}$ & $25.174 \mu \mathrm{M}$ & $2.10 \times 10^{-7}$ & 1.17 \\
$\mathrm{Mn}^{2+}$ & $5.557 \mu \mathrm{M}$ & $2.10 \times 10^{-7}$ & 1.04 \\
$\mathrm{Cu}^{2+}$ & $1.602 \mu \mathrm{M}$ & $2.10 \times 10^{-7}$ & 1.01 \\
$\mathrm{Co}^{2+}$ & $0.859 \mu \mathrm{M}$ & $2.10 \times 10^{-7}$ & 1.00 \\
& & total product of cation & 44100 \\
& & exchange reduction & \\
& & factors: &
\end{tabular}

data of cation exchange studies ${ }^{84,85}$ and was also used for the other divalent cations. Finally, Table 2 lists the calculated cation exchange reduction factors of the localized surface proton concentration for $\mathrm{pH}_{\mathrm{pB}}=10.5$. The total product of these factors (total cation exchange reduction factor in the denominator of eq $7)$ is a large number $(44,100)$ compared to unity, indicating the very important role of cation-proton exchange at the membrane surface in controlling the localized surface proton concentration.

As reported previously in ref 6, the localized proton population density $\left[\mathrm{H}_{\mathrm{L}}^{+}\right]^{0}$ before the cation-proton exchange process was calculated to be $24.6 \mathrm{mM}$ when the membrane electrical potential difference potential was $180 \mathrm{mV}$. In this example, the localized proton concentration $\left[\mathrm{H}_{\mathrm{L}}^{+}\right]$is reduced apparently by the cation exchange to $24.6 \mathrm{mM} / 44100=5.58 \times$ $10^{-7} \mathrm{M}$, which is equivalent to a localized proton $\mathrm{pH}$ of $6.25-$ remarkably lower than the bulk liquid culture $\mathrm{pH}$ of 10.5 .

\section{ASSOCIATED CONTENT}

\section{SI Supporting Information}

The Supporting Information is available free of charge at https://pubs.acs.org/doi/10.1021/acsomega.0c01768.

Localized proton concentrations at the liquid-membrane interface, protonic motive forces, and Gibbs free energy (XLSX)

\section{AUTHOR INFORMATION}

\section{Corresponding Author}

James Weifu Lee - Department of Chemistry and Biochemistry, Old Dominion University, Norfolk, Virginia 23529, United States; @orcid.org/0000-0003-2525-5870; Email: jwlee@ odu.edu

Complete contact information is available at: https://pubs.acs.org/10.1021/acsomega.0c01768

\section{Author Contributions}

J.W.L. designed and performed the research, analyzed the data, and wrote the paper.

\section{Notes}

The author declares no competing financial interest.

\section{ACKNOWLEDGMENTS}

The author wishes to thank Drs. William H. Schlesinger, Gerald H. Pollack, Tobias Baskin, Todd P. Silverstein, Peter Rich, David G. Nicholls, Peter Pohl, and Patrick G. Hatcher for their discussions. This work was supported in part with the Lee laboratory start-up research funds provided by the Department of Chemistry and Biochemistry, the College of Sciences, the Office of Research at Old Dominion University, and by the Old Dominion University Research Foundation. The author declares no competing interests. All data is available in the main text and the support information (xlsx efile of excel spreadsheet showing how the protonic motive forces and the associated Gibbs free energy were calculated).

\section{REFERENCES}

(1) Pisano, R.; Anakkar, A.; Pellegrino, E. M.; Nagels, M. Thermodynamic foundations of physical chemistry: reversible processes and thermal equilibrium into the history. Found. Chem. 2019, 21, 297-323.

(2) Krulwich, T. A. Bioenergetics of alkalophilic bacteria. J. Membr. Biol. 1986, 89, 113-125.

(3) Krulwich, T. A.; Guffanti, A. A. Alkalophilic bacteria. Annu. Rev. Microbiol. 1989, 43, 435-463.

(4) Olsson, K.; Keis, S.; Morgan, H. W.; Dimroth, P.; Cook, G. M. Bioenergetic properties of the thermoalkaliphilic Bacillus sp. strain TA2. A1. J. Bacteriol. 2003, 185, 461-465.

(5) Lee, J. W. Proton-electrostatics hypothesis for localized proton coupling bioenergetics. Bioenergetics 2012, 01, 1-8.

(6) Lee, J. Proton-electrostatic localization: explaining the bioenergetic conundrum in alkalophilic bacteria. Bioenergetics 2015, 4, 1-8.

(7) Saeed, H.; Lee, J. Experimental determination of proton-cation exchange equilibrium constants at water-membrane interface fundamental to bioenergetics. WATER J.: Multidiscip. Res. J. 2018, 9, 116140

(8) Lee, J. W. A possible electrostatic interpretation for proton localization and delocalization in chloroplast bioenergetics system. Biophys. J. 2005, 88, 324a-325a.

(9) Lee, J. W. Electrostatically localized proton bioenergetics: better understanding membrane potential. Heliyon 2019, 5, No. e01961.

(10) Mitchell, P. Coupling of phosphorylation to electron and hydrogen transfer by a chemi-osmotic type of mechanism. Nature 1961, 191, 144-148.

(11) Mitchell, P. Possible molecular mechanisms of the protonmotive function of cytochrome systems. J. Theor. Biol. 1976, 62, 327-367.

(12) Mitchell, P. David Keilin's respiratory chain concept and its chemiosmotic consequences. Nobel prize lect. 1978, 1, 295-330.

(13) Garrett, R. H.; Grisham, C. M. Biochemistry, Brooks; 5 ed.; 2013.

(14) Nelson, D. L.; Lehninger, A. L.; Cox, M. M. Lehninger principles of biochemistry; 6 ed.; Macmillan: 2013.

(15) Nicholls, D. G.; Ferguson, S. J. 3-QUANTITATIVE BIOENERGETICS: THE MEASUREMENT OF DRIVING FORCES. In Bioenergetics 2, Ferguson, D. G. N. J., Ed. Academic Press: 1992; pp $38-$ 63.

(16) Nicholls, D. G.; Ferguson, S. J. Quantitative Bioenergetics. In Bioenergetics; (4th Edition), Academic Press: Boston, 2013; pp 27-51.

(17) Mitchell, P. The Correlation of Chemical and Osmotic Forces in Biochemistry. J. Biochem. 1985, 97, 1-18.

(18) Mitchell, P.; Moyle, J. Estimation of Membrane Potential and Ph Difference across the Cristae Membrane of Rat Liver Mitochondria. Eur. J. Biochem. 1969, 7, 471-484. 
(19) Saeed, H. A.; Lee, J. W. Experimental Demonstration of Localized Excess Protons at a Water-Membrane Interface. Bioenergetics 2015, 04, 1-7.

(20) Lee, J. Localized excess protons and methods of making and using the same. United States Patent Application Publication No. US 20170009357 A1 2017, (Copyright (C) 2017 American Chemical Society (ACS). All Rights Reserved.), 73pp.

(21) Sturr, M. G.; Guffanti, A. A.; Krulwich, T. A. Growth and bioenergetics of alkaliphilic Bacillus firmus OF4 in continuous culture at high pH. J. Bacteriol. 1994, 176, 3111-3116.

(22) Krulwich, T. A. Alkaliphiles:'basic'molecular problems of $\mathrm{pH}$ tolerance and bioenergetics. Mol. Microbiol. 1995, 15, 403-410.

(23) Padan, E.; Bibi, E.; Ito, M.; Krulwich, T. A. Alkaline pH homeostasis in bacteria: new insights. Biochim. Biophys. Acta, (BBA)biomembranes 2005, 1717, 67-88.

(24) Guffanti, A. A.; Krulwich, T. A. Bioenergetic problems of alkalophilic bacteria. Biochem. Soc. Trans. 1984, 12, 411.

(25) Krulwich, T. A.; Gilmour, R.; Hicks, D. B.; Guffanti, A. A.; Ito, M. Energetics of alkaliphilic Bacillus species: physiology and molecules. Adv. Microb. Physiol. 1998, 40, 401-438.

(26) Krulwich, T. A.; Liu, J.; Morino, M.; Fujisawa, M.; Ito, M.; Hicks, D. B. Adaptive mechanisms of extreme alkaliphiles. Extremophiles handb. 2011, 119-139.

(27) Vinkler, C.; Avron, M.; Boyer, P. D. Initial formation of ATP in photophosphorylation does not require a proton gradient. FEBS Lett. 1978, 96, 129-134.

(28) Ferguson, S. J. Fully delocalised chemiosmotic or localised proton flow pathways in energy coupling?: A scrutiny of experimental evidence. Biochim. Biophys. Acta, (BBA)-Rev. Bioenerg. 1985, 811, 4795.

(29) Dilley, R. A.; Theg, S. M.; Beard, W. A. Membrane-proton interactions in chloroplast bioenergetics: Localized proton domains. Annual review of plant physiology 1987, 38, 347-389.

(30) Dilley, R. A. On why thylakoids energize ATP formation using either delocalized or localized proton gradients-a Ca2+ mediated role in thylakoid stress responses. Photosynth. Res. 2004, 80, 245-263.

(31) Williams, R. J. P. Chemical advances in evolution by and changes in use of space during time. J. Theor. Biol. 2011, 268, 146-159.

(32) Williams, R. J. P. The Multifarious Couplings of Energy Transduction. Biochim. Biophys. Acta 1978, 505, 1-44.

(33) Williams, R. J. Proton-Driven Phosphorylation Reactions in Mitochondrial and Chloroplast Membranes. FEBS Lett. 1975, 53, 123125.

(34) Williams, R. J. P.; et al. J. Theor. Biol. 1961, 1, 1-7.

(35) Chiang, G. G.; Dilley, R. A. Intact Chloroplasts Show Ca ${ }^{2+}$-Gated Switching between Localized and Delocalized Proton Gradient Energy Coupling (Atp Formation). Plant Physiol. 1989, 90, 1513-1523.

(36) Xiong, J.-W.; Zhu, L.; Jiao, X.; Liu, S.-S. Evidence for $\Delta \mathrm{pH}$ surface component $\left(\Delta \mathrm{pH}^{\mathrm{S}}\right)$ of proton motive force in ATP synthesis of mitochondria. Biochim. Biophys. Acta, Gen. Subj. 2010, 1800, 213-222.

(37) Rieger, B.; Junge, W.; Busch, K. B. Lateral pH gradient between OXPHOS complex IV and $\mathrm{F}_{0} \mathrm{~F}_{1}$ ATP-synthase in folded mitochondrial membranes. Nat. Commun. 2014, 5, 3103.

(38) Weichselbaum, E.; Österbauer, M.; Knyazev, D. G.; Batishchev, O. V.; Akimov, S. A.; Nguyen, T. H.; Zhang, C.; Knör, G.; Agmon, N.; Carloni, P.; Pohl, P. Origin of proton affinity to membrane/water interfaces. Sci. Rep. 2017, 7, 4553.

(39) Lee, J. W. Localized excess protons and methods of making and using same. No. US 10,501,854 B2, 2019.

(40) Lee, J., Localized excess protons and methods of making and using the same. PCT International Patent Application Publication No. WO 2017/007762 A1, 2017, 56 pages.

(41) McLaughlin, S. The Electrostatic Properties of Membranes. Annu. Rev. Biophys. Biophys. Chem. 1989, 18, 113-136.

(42) Grahame, D. C. The electrical double layer and the theory of electrocapillarity. Chem. Rev. 1947, 41, 441-501.

(43) Lee, J. W. Membrane surface charges attracted protons are not relevant to proton motive force. Bioenergetics 2013, 02, No. e114.
(44) Lee, J. W. Protonic Capacitor: Elucidating the biological significance of mitochondrial cristae formation. Sci. Rep. 2020, 10304.

(45) Preiss, L.; Klyszejko, A. L.; Hicks, D. B.; Liu, J.; Fackelmayer, O. J.; Yildiz, Ö.; Krulwich, T. A.; Meier, T. The c-ring stoichiometry of ATP synthase is adapted to cell physiological requirements of alkaliphilic Bacillus pseudofirmus OF4. Proc. Natl. Acad. Sci. U. S. A. 2013, 110, 7874-7879.

(46) Preiss, L.; Langer, J. D.; Hicks, D. B.; Liu, J.; Yildiz, Ö.; Krulwich, T. A.; Meier, T. The c-ring ion binding site of the ATP synthase from B acillus pseudofirmus OF 4 is adapted to alkaliphilic lifestyle. Mol. Microbiol. 2014, 92, 973-984.

(47) Boyer, P. D. A research journey with ATP synthase. J. Biol. Chem. 2002, 277, 39045-39061.

(48) Boyer, P. D. Energy, life, and ATP. Biosci. Rep. 1998, 18, 97-117.

(49) Gu, J.; Wu, M.; Guo, R.; Yan, K.; Lei, J.; Gao, N.; Yang, M. The architecture of the mammalian respirasome. Nature 2016, 537, 639643.

(50) Dudkina, N. V.; Kouřil, R.; Peters, K.; Braun, H.-P.; Boekema, E. J. Structure and function of mitochondrial supercomplexes. Biochim. Biophys. Acta, Bioenerg. 2010, 1797, 664-670.

(51) Kühlbrandt, W. Structure and function of mitochondrial membrane protein complexes. BMC Biol. 2015, 13, 89.

(52) Davies, K. M.; Strauss, M.; Daum, B.; Kief, J. H.; Osiewacz, H. D.; Rycovska, A.; Zickermann, V.; Kühlbrandt, W. Macromolecular organization of ATP synthase and complex I in whole mitochondria. Proc. Natl. Acad. Sci. U. S. A. 2011, 108, 14121-14126.

(53) Davies, K. M.; Anselmi, C.; Wittig, I.; Faraldo-Gómez, J. D.; Kühlbrandt, W. Structure of the yeast $\mathrm{F}_{1} \mathrm{~F}_{\mathrm{o}}$-ATP synthase dimer and its role in shaping the mitochondrial cristae. Proc. Natl. Acad. Sci. U. S. A. 2012, 109, 13602-13607.

(54) Davies, K. M.; Daum, B. Role of cryo-ET in membrane bioenergetics research. Biochem. Soc. Trans. 2013, 41, 1227-1234.

(55) Althoff, T.; Mills, D. J.; Popot, J.-L.; Kühlbrandt, W. Arrangement of electron transport chain components in bovine mitochondrial supercomplex $\mathrm{I}_{1} \mathrm{III}_{2} \mathrm{IV}_{1}$. EMBO J. 2011, 30, 4652-4664.

(56) Daum, B.; Walter, A.; Horst, A.; Osiewacz, H. D.; Kühlbrandt, W. Age-dependent dissociation of ATP synthase dimers and loss of innermembrane cristae in mitochondria. Proc. Natl. Acad. Sci. U. S. A. 2013, 110, 15301-15306.

(57) Letts, J. A.; Fiedorczuk, K.; Sazanov, L. A. The architecture of respiratory supercomplexes. Nature 2016, 537, 644-648.

(58) Saeed, H. Bioenergetics: Experimental Demonstration of Excess Protons and Related Features. PhD Thesis, Old Dominion University, Norfolk, VA 23529 USA 2016.

(59) Cao, F. J.; Feito, M. Thermodynamics of feedback controlled systems. Phys. Rev. E 2009, 79, No. 041118.

(60) Cao, F. J.; Feito, M.; Touchette, H. Information and flux in a feedback controlled Brownian ratchet. Phys. A 2009, 388, 113-119.

(61) Lee, J. W. (Zhejiang Agriculture University, D. o. A., Student Research Group on Thermotroph Exploration Study led by Lee), There may be thermotrophic type of life on Earth; Potential Science, published in Beijing, China 1983, 1.

(62) Nikulov, A. Observations of Persistent Current at Non-Zero Resistance: Challenge to the Second Law of Thermodynamics. AIP Conf. Proc. 2011, 1411, 122.

(63) Sheehan, D. P. Infrared Cloaking, Stealth, and the Second Law of Thermodynamics. Entropy 2012, 14, 1915-1938.

(64) Sheehan, D. P. Maxwell Zombies: Conjuring the Thermodynamic Undead. Am. Sci. 2018, 106, 234-241.

(65) Sheehan, D. P.; Garamella, J. T.; Mallin, D. J.; Sheehan, W. F. Steady-state nonequilibrium temperature gradients in hydrogen gasmetal systems: challenging the second law of thermodynamics. Phys. Scr. 2012, T151, No. 014030.

(66) Pal, P. S.; Rana, S.; Saha, A.; Jayannavar, A. M. Extracting work from a single heat bath: A case study of a Brownian particle under an external magnetic field in the presence of information. Phys. Rev. E 2014, 90, No. 022143. 
(67) Sheehan, D. P.; Mallin, D. J.; Garamella, J. T.; Sheehan, W. F. Experimental Test of a Thermodynamic Paradox. Found. Phys. 2014, $44,235-247$.

(68) Serreli, V.; Lee, C.-F.; Kay, E. R.; Leigh, D. A. A molecular information ratchet. Nature 2007, 445, 523-527.

(69) Vologodskii, A. V.; Zhang, W.; Rybenkov, V. V.; Podtelezhnikov, A. A.; Subramanian, D.; Griffith, J. D.; Cozzarelli, N. R. Mechanism of topology simplification by type II DNA topoisomerases. Proc. Natl. Acad. Sci. U. S. A. 2001, 98, 3045-3049.

(70) Jennings, R. C.; Belgio, E.; Zucchelli, G. Photosystem I, when excited in the chlorophyll $\mathrm{Q}(\mathrm{y})$ absorption band, feeds on negative entropy. Biophys. Chem. 2018, 233, 36-46.

(71) Lee, J. W. Isothermal electricity for energy renewal PCT International Patent Application Publication Number WO 2019/136,037 A1 2019.

(72) Koski, J. V.; Maisi, V. F.; Pekola, J. P.; Averin, D. V. Experimental realization of a Szilard engine with a single electron. Proc. Natl. Acad. Sci. U. S. A. 2014, 111, 13786-13789.

(73) Battail, G. Living Versus Inanimate: The Information Border. Biosemiotics 2009, 2, 321-341.

(74) Lehninger, A. L.; Nelson, D. L.; Cox, M. M. Lehninger Principles of Biochemistry; 6th ed.; Macmillan: New York, 2013.

(75) Garrett, R.; Grisham, C. Biochemistry textbook; 5th ed.; Brooks/ Cole CENGAGE Learning: United States of America, 2013.

(76) Marx, D.; Tuckerman, M. E.; Hutter, J.; Parrinello, M. The nature of the hydrated excess proton in water. Nature 1999, 397, 601-604.

(77) Pomès, R.; Roux, B. Molecular mechanism of $\mathrm{H}^{+}$conduction in the single-file water chain of the gramicidin channel. Biophys. J. 2002, 82, 2304-2316.

(78) Marx, D. Proton transfer 200 years after von Grotthuss: Insights from ab initio simulations. ChemPhysChem 2006, 7, 1848-1870.

(79) Zheng, Y.; Shojaei-Baghini, E.; Wang, C.; Sun, Y. Microfluidic characterization of specific membrane capacitance and cytoplasm conductivity of single cells. Biosens. Bioelectron. 2013, 42, 496-502.

(80) Azzone, G.; Benz, R.; Bertl, A.; Colombini, M.; Crofts, A.; Dilley, R.; Dimroth, P.; Dutton, P. L.; Felle, H.; Harold, F.; Junge, W.; Kaback, H. R.; Knaff, D.; Krulwich, T.; Lodish, H.; Malmström, B.; Maloney, P.; Mannella, C.; Padan, E.; Papa, S.; Rottenberg, H.; Rudnick, G.; Rydström, J.; Silverstein, T.; Skulachev, V.; Slayman, C.; Tedeschi, H.; Wikström, M.; Wilson, T. H. Transmembrane Measurements across Bioenergetic Membranes. Biochim. Biophys. Acta 1993, 1183, $1-3$.

(81) Bertl, A.; Blumwald, E.; Coronado, R.; Eisenberg, R.; Findlay, G.; Gradmann, D.; Hille, B.; Kohler, K.; Kolb, H.-A.; MacRobbie, E.; Meissner, G.; Miller, C.; Neher, E.; Palade, P.; Pantoja, O.; Sanders, D.; Schroeder, J.; Slayman, C.; Spanswick, R.; Walker, A.; Williams, A. Electrical Measurements on Endomembranes. Science 1992, 258, 873874.

(82) Guffanti, A. A.; Hicks, D. B. Molar growth yields and bioenergetic parameters of extremely alkaliphilic Bacillus species in batch cultures, and growth in a chemostat at $\mathrm{pH}$ 10.5. J. Gen. Microbiol. 1991, 137, $2375-2379$.

(83) Guffanti, A. A.; Susman, P.; Blanco, R.; Krulwich, T. A. Protonmotive Force and $\alpha$-Aminoisobutyric-Acid Transport in an Obligately Alkalophilic Bacterium. J. Biol. Chem. 1978, 253, 708-715.

(84) Lee, J. W.; Kidder, M.; Evans, B. R.; Paik, S.; Buchanan, A. C., III; Garten, C. T.; Brown, R. C. Characterization of Biochars Produced from Cornstovers for Soil Amendment. Environ. Sci. Technol. 2010, 44, 7970-7974.

(85) Skjemstad, J. O.; Gillman, G. P.; Massis, A.; Spouncer, L. R. Measurement of Cation Exchange Capacity of Organic-Matter Fractions from Soils using a Modified Compulsive Exchange Method. Commun. Soil Sci. Plant Anal. 2008, 39, 926-937. 